\title{
Nonlinear morphological control of growing crystals
}

\author{
Shuwang Li ${ }^{\mathrm{a}, \mathrm{b}}$, John S. Lowengrub ${ }^{\mathrm{b}, *}$, Perry H. Leo ${ }^{\mathrm{a}}$ \\ ${ }^{a}$ Department of Aerospace Engineering and Mechanics, University of Minnesota, Minneapolis, MN 55455, USA \\ ${ }^{\mathrm{b}}$ Department of Mathematics, University of California at Irvine, Irvine, CA 92697, USA
}

Received 11 February 2005; received in revised form 11 February 2005; accepted 19 June 2005

Available online 14 July 2005

Communicated by H. Levine

\begin{abstract}
In this paper, we study the nonlinear stability of growing crystals and the development of protocols for controlling their shapes. We focus on the effects of surface tension anisotropy on the morphology of a $2 \mathrm{D}$, non-circular crystal growing in a supercooled melt. We use a spectrally accurate boundary integral method to simulate the long-time, nonlinear dynamics of evolving crystals and to characterize the nonlinear morphological stability during growth. Our analysis and simulations reveal that under critical conditions of an applied far-field heat flux, there is nonlinear stabilization leading to the growth of compact crystals even though unstable growth may be significant at early times. The crystal morphologies are determined by a complex competition between the heat transport (far-field flux) and the strength of surface tension anisotropy. In particular, we observe three types of behavior: universal, limiting and oscillatory. Universal behavior occurs when the shape of the evolving crystal tends to a shape that is independent of both time and the initial condition. Limiting behavior occurs when the shape of the crystal tends to a shape that is independent of time, but depends on the initial condition. Oscillatory behavior occurs when the shape depends on both time and the initial condition, though its growth is bounded. Unlike the isotropic case where universal shapes have an arbitrary symmetry that depends only on the far-field flux [S. Li, J. Lowengrub, P. Leo, V. Cristini, Nonlinear Stability Analysis of Self-similar crystal growth: control of Mullins-Sekerka instability, J. Crystal Growth 277 (2005) 578-592.], here the surface tension anisotropy selects the symmetry of the universal shape. The results are presented as a phase diagram that characterizes the long time evolution behavior in a plane parameterized by the far-field flux and the strength of surface tension anisotropy. The phase diagram delineates the parameter ranges for the observed behaviors and can be used to design a nonlinear protocol to control the shapes of growing crystals that might be able to be carried out in an experiment. Finally, the analysis developed here may provide insights on the control of pattern formation in other physical and biological systems.
\end{abstract}

(c) 2005 Elsevier B.V. All rights reserved.

Keywords: Crystal growth; Mullins-Sekerka instability; Self-similar; Diffusion; Morphological stability; Pattern formation; Anisotropy

\footnotetext{
* Corresponding author.

E-mailaddresses: sli@aem.umn.edu (S. Li); lowengrb@math.uci.edu (J.S. Lowengrub); phleo@aem.umn.edu (P.H. Leo).
} 


\section{Introduction}

Pattern formation is typically characterized by a competition among stabilizing and destabilizing effects (e.g. [1]). This is particularly evident for crystal growth problems where it has been recognized that growth morphologies are determined by the interaction between macroscopic driving forces (applied supercooling or far-field flux) and microscopic interfacial forces (surface tension, kinetics of atomic attachment, anisotropies, etc.) and their associated time scales. The interaction among these forces produces complex morphologies including dendrites and side-branches, which have been extensively studied theoretically (e.g. [2-18]) and experimentally (e.g. [26-31]). The complex morphologies are a result of the Mullins-Sekerka instability. In many applications (e.g. castings) it is desirable to suppress the instability and prevent the formation of complex shapes. Mullins and Sekerka [15] identified the possibility of growing crystals with compact shapes when the supercooling is kept sub-critical but this was not quantified. Much less work has been performed on this topic. Our work here helps to fill this gap. More generally, our work also may provide insights on the control of pattern formation in other physical and biological systems such as epitaxial growth of thin films (e.g. [19]), viscous fingering (e.g. [20]), bacteria colonies (e.g. [21]) and tumor growth (e.g. [22-24]).

The Mullins-Sekerka instability arises because of the imbalance of time scales governing the macroscopic and microscopic forces. For example, the ratio of the time scale for growth $t_{\infty}$ (due to a constant supercooling) and the time scale over which surface tension acts $t_{\tau}$ scales as $t_{\infty} / t_{\tau} \propto R^{-1}$ where $R$ is the effective radius of the crystal, i.e. radius of a sphere with the same volume (see [2,12]). Thus, the surface tension has less and less time to stabilize the shape during growth. In 3D, Cristini and Lowengrub [2,3] showed that the time scales $t_{\infty}$ and $t_{\tau}$ can be balanced (i.e. $\tau_{\infty} / t_{\tau} \propto 1$ ) and the Mullins-Sekerka instability can be suppressed if instead the far-field heat flux is constant (the supercooling is time dependent). Note that what we mean by flux here is the integral flux applied at the far-field boundary, see Eq. (4). In particular, Cristini and Lowengrub [3] showed numerically via 3D adaptive boundary integral simulations that compact nonlinear crystal shapes can be achieved. Their results also suggested that nonlinear self-similar evolution and the control of crystal morphologies are possible.

In $2 \mathrm{D}$, in order to balance the growth time and surface tension time scales and to suppress the MullinsSekerka instability, the far-field heat flux needs to be scaled as $R^{-1}$ because of the spatial differences between the 2D and 3D growth [4,5,12]. Li et al. [4] identified precise heat flux conditions such that these two time scales balance. They developed a nonlinear theory of self-similar crystal growth and demonstrated the existence of 2D non-circular self-similar solutions [4]. Recently, Li et al. [5] performed a stability analysis of these self-similar solutions under isotropic surface tension. Very interestingly, at long times nonlinear stabilization was found even though unstable growth may be significant at early times. This stabilization was found to lead to the existence of universal limiting shapes. In particular, the morphologies of the nonlinearly evolving crystals tend to limiting shapes that evolve self-similarly and depend only on the flux [5]. A number of limiting shapes exist for each flux (the number of possible shapes actually depends on the flux), but only one is universal in the sense that a crystal with an arbitrary initial shape will evolve to this universal shape. Linear theory was found to provide a good prediction for the flux to achieve a $k$-fold symmetric universal shape. Oscillatory behavior may occur for a narrow range of fluxes near the transition region from $k$-fold to $(k+1)$ fold symmetric universal shapes. A phase diagram was constructed to characterize the achievable symmetries of the limiting shapes as a function of the far-field flux.

In this paper, we extend our recent study [5] and examine the effects of surface tension anisotropy on the morphology of a 2D, non-circular crystal growing in a supercooled melt. We use a spectrally accurate boundary integral method to simulate the long-time, nonlinear dynamics of evolving crystals and to characterize the nonlinear morphological stability during growth. Our analysis and simulations reveal that under critical conditions of an applied far-field heat flux, there is nonlinear stabilization leading to the growth of compact crystals even though unstable growth may be significant at early times. The crystal morphologies are determined by a complex competition between 
the heat transport (far-field flux) and the strength of surface tension anisotropy. In particular, we observe three types of behavior: universal, limiting and oscillatory. Universal behavior occurs when the shape of the evolving crystal tends to a shape that is independent of both time and the initial condition. Limiting behavior occurs when the shape of the crystal tends to a shape that is independent of time, but depends on the initial condition. Oscillatory behavior occurs when the shape depends on both time and the initial condition, though its growth is bounded. Unlike the isotropic case where universal shapes have an arbitrary symmetry that depends only on the far-field flux [5], here the surface tension anisotropy selects the symmetry of the universal shape. The results are presented as a phase diagram that characterizes the long time evolution behavior in a plane parameterized by the far-field flux and the strength of surface tension anisotropy. The phase diagram delineates the parameter ranges for the observed behaviors and can be used to design a nonlinear protocol to control the shapes of growing crystals that might be able to be carried out in an experiment.

Although this paper focuses on crystal growth in 2D, our analysis applies qualitatively to $3 \mathrm{D}$. This is because once the difference between 2D and 3D heat fluxes (i.e. area versus volume growth) is scaled out the governing equations are very similar [4]. In addition, the linear analysis in [5] shows that interactions among the perturbation modes are similar in both 2D and 3D. However, the surface tension anisotropy is more complicated in $3 \mathrm{D}$, since there are two principal directions of curvature.

We note that in the special case in which the velocity of the crystal is determined locally by the surface energy, and not by diffusion as considered here, it has been shown that the evolution tends to the Wulff shape [35,36]. The relevance of this example to our work is currently under study.

This paper is organized as follows: in Section 2 , we review the governing equations, and linear stability analysis; in Section 3, we describe the numerical scheme; in Section 4, we briefly review the results of a crystal with isotropic surface tension. in Section 5, we discuss numerical results; and in Section 6, we give conclusions and describe work in progress.

\section{Theory}

\subsection{Governing equations}

We consider a two-dimensional solid crystal growing quasi-statically in a supercooled liquid phase. The interface $\Sigma$ separates the solid phase $\Omega_{1}$ from the liquid phase $\Omega_{2}$. We assume for simplicity that local equilibrium holds at the interface, and the thermal diffusivities of the two phases are identical. However, the results presented herein apply more generally [37]. Using the nondimensionalization given in $[2,3]$ in which the length scale is the equivalent radius of the crystal at time $t=0$ and the time scale is the characteristic surface tension relaxation time scale, the following equations govern the growth of the crystal:

$$
\begin{aligned}
& \nabla^{2} T_{i}=0 \quad \text { in } \Omega_{i} \quad i=1,2 \\
& V=\left(\nabla T_{1}-\nabla T_{2}\right) \cdot \mathbf{n} \quad \text { on } \Sigma \\
& T_{1}=T_{2}=-\tau(\mathbf{n}) \kappa \quad \text { on } \Sigma \\
& J=-\frac{1}{2 \pi} \lim _{R_{\infty} \rightarrow \infty} \int_{\Sigma_{\infty}} \nabla T_{2} \cdot \mathbf{n} \mathrm{d} \Sigma_{\infty}=\frac{1}{2 \pi} \int_{\Sigma} V \mathrm{~d} s
\end{aligned}
$$

where $\Sigma_{\infty}$ is a circle with radius $R_{\infty}$. The interface $\Sigma$ evolves via

$\mathbf{n} \cdot \frac{\mathrm{d} \mathbf{x}}{\mathrm{d} t}=V \quad$ on $\Sigma$,

where $T_{i}$ is the temperature field, $i=1$ for solid phase and $i=2$ for liquid phase, $V$ is the normal velocity of the interface, $\mathbf{n}$ is the unit normal directed towards $\Omega_{2}, \kappa$ is the curvature, $\tau$ is the anisotropic surface tension, and $J$ is the far-field heat flux. In Eq. (4) we have used the fact that the $T_{i}$ are harmonic so that $J$ specifies the time derivative of the area of the solid phase. In 2D, the normal vector $\mathbf{n}=\left(n_{1}, n_{2}\right)$ can be characterized in terms of the tangent angle $\theta=\tan ^{-1}\left(-n_{1} / n_{2}\right)$, i.e. the angle that a tangent vector makes with the $x_{1}$-axis. Thus, the anisotropic surface tension $\tau=\tau(\theta)$. Further, $\tau(\theta)=\gamma(\theta)+\gamma^{\prime \prime}(\theta)$ where $\gamma(\theta)$ is the anisotropic surface energy. For a general $m$-fold symmetric anisotropy,

$$
\begin{aligned}
& \gamma(\theta)=1+v_{m} \cos m \theta, \\
& \tau(\theta)=1-\left(m^{2}-1\right) v_{m} \cos m \theta
\end{aligned}
$$


where $v_{m}$ is the strength of the anisotropy (e.g. see [14]). In this paper, we restrict our study to the case $v_{m}<1 /\left(m^{2}-1\right)$. Thus, there are no missing orientations, as would be the case with larger anisotropies.

\subsection{Linear analysis}

In this section, we analyze the linear stability of a growing crystal following $[15,16,2,4,25]$. We take the perturbed crystal-melt interface to be given by a linear combination of Fourier modes,

$r(\alpha, t)=R(t)+\sum_{k=2}^{\infty} \delta_{k}(t) \cos k \alpha$,

where $R(t)$ is the radius of a underlying growing circle $(R(0)=1)$, the $\delta$ 's are amplitudes of perturbations, and $\alpha$ is the polar angle. We assume that the anisotropy $v_{m}$ is on the same order as the amplitudes of perturbations so that products of $v_{m}$ and $\delta$ 's are neglected. Note that in Eq. (7), $k=1$ means a translation of a circle (i.e. no shape perturbation occurs). The rate of area growth for the unperturbed circle is

$R(t) \frac{\mathrm{d} R}{\mathrm{~d} t}=J(t)$,

where $J(t)$ is the specified flux. The far-field temperature $T_{\infty}$ is related to the heat flux via

$T_{\infty}(t)=-J(t) \log \left(\frac{R_{\infty}}{R(t)}\right)-\frac{1}{R(t)}$,

where $R_{\infty}$ is the radius of a large circle containing the growing crystal [4]. The growth rate of the $k$-th mode perturbation,

$$
\left(\frac{\delta_{k}}{R}\right)^{-1} \frac{\mathrm{d}}{\mathrm{d} t}\left(\frac{\delta_{k}}{R}\right)=\frac{(k-2)\left(J-J_{k}\right)}{R^{2}},
$$

where the critical flux $J_{k}$ and the associated linear flux constant $C_{k}$ are

$$
\begin{aligned}
J_{k}(t) & =\frac{C_{k}}{R(t)}, \\
C_{k} & =\frac{2 k\left(k^{2}-1\right)}{k-2} \cdot \begin{cases}1-\frac{v_{m}}{\left(\delta_{m}(t) / R(t)\right)} & k=m, \\
1 & k \neq m .\end{cases}
\end{aligned}
$$

If the applied flux $J<J_{k}(t)$, then the $k$-th mode perturbation decays. If $J>J_{k}(t)$, the $k$-th mode perturbation grows. If $J=J_{k}(t)$ the $k$-th mode perturbation is unchanged (i.e. self-similar [4]). As discussed in Section 1 , the critical flux $J_{k}(t)$ scales as $1 / R(t)$ in order to balance the time scales for growth and surface tension. Also, because of anisotropy $J_{k}(t)$ depends on the current shape through $\delta_{k}(t) / R(t)$ when $k=m$. Note that that anisotropy reduces the critical flux compared to the isotropic case where $v_{m}=0$, see Eq. (11).

It can be shown [37] that by taking an alternative flux

$$
\begin{aligned}
\tilde{J}_{k}(t) & =\frac{C_{k}}{R(t)}, \\
\tilde{C}_{k} & =\frac{2 k\left(k^{2}-1\right)}{k-2} \cdot \begin{cases}1-\frac{v_{m}}{\left(\delta_{m} / R\right)} & k=m, \\
1 & k \neq m,\end{cases}
\end{aligned}
$$

where $\left(\delta_{m} / R\right)_{\infty} \geq v_{m}$ is a time independent parameter then the evolution of $\delta_{m} / R$ tends to $\left(\delta_{m} / R\right)_{\infty}$ as $R \rightarrow \infty$ if $J=\tilde{J}_{m}$ for any initial perturbation $\delta_{m}(0) / R(0)$. Note that if $\left(\delta_{m} / R\right)_{\infty}<v_{m}$, then the crystal shrinks and $\delta_{m} / R$ tends to $\left(\delta_{m} / R\right)_{\infty}$ as $R \rightarrow 0$. In contrast, in the isotropic case $v_{m}=0$ the initial perturbation is unchanged in time, i.e. $\delta_{m}(t) / R(t)=\delta_{m}(0) / R(0)$ if $J=\tilde{J}_{m}$. Thus, the presence of anisotropy provides a mechanism to control the growth of mode $m$. At the level of linear theory, modes $k \neq m$ evolve as in the isotropic case.

If the initial shape of an interface is a mixture of Fourier modes, the flux that makes $l$-th mode have the largest growth rate can be derived as

$$
J_{l}^{*}(t)=\frac{6 l^{2}-2}{R(t)} \cdot \begin{cases}1-\frac{v_{m}}{\left(\delta_{m}(t) / R(t)\right)} l & =m, \\ 1 & l \neq m .\end{cases}
$$

Given a wavenumber $l \neq m$ and taking the applied flux to be $J=J_{l}^{*}$, then Eq. (10) can be solved to yield:

$$
\frac{\delta_{k}}{R}(t)= \begin{cases}\frac{2 m\left(m^{2}-1\right) v_{m}}{-q(m, l)\left(6 l^{2}-2\right)}+\beta_{m} R(t)^{q(m, l)} & k=m, \\ \beta_{k} R(t)^{q(k, l)} & k \neq m,\end{cases}
$$

where $k$ is an arbitrary mode and $q(k, l)$ is given by

$q(k, l)=\frac{-2 k\left(k^{2}-1\right)+(k-2)\left(6 l^{2}-2\right)}{\left(6 l^{2}-2\right)}$, 
and

$\beta_{m}=\frac{\delta_{m}}{R}(0)+\frac{2 m\left(m^{2}-1\right) v_{m}}{q(m, l)\left(6 l^{2}-2\right)} \quad$ and $\quad \beta_{k}=\frac{\delta_{k}}{R}(0)$.

Eq. (14) shows that the anisotropy symmetry mode $m$ associated with the surface tension can compete with mode $l$, the mode with the fastest growth rate. For instance, there exists a critical radius $R_{\text {lin }}^{*}$ such that the growth rates of mode $m$ and $l$ are equal at $R_{l i n}^{*}$,

$R_{\text {lin }}^{*}=\left(\frac{q(m, l) \beta_{m}}{q(l, l) \beta_{l}}\right)^{\frac{1}{p}}$,

where $p=q(l, l)-q(m, l)$. For $R<R_{\text {lin }}^{*}$, the mode $m$ grows faster than mode $l$ even though $J=J_{l}^{*}$. For $R \geq R_{\text {lin }}^{*}$ mode $l$ dominates.

In addition, Eq. (14) shows that if $l<m$ and $q<0$ then $\delta_{m} / R$ tends to a finite value that depends only on $v_{m}, m, l$ and is independent of the initial data. If in addition $l<k$ and $q<0$, for any $k$, or if only modes $k>m$ are present initially the linear evolution converges to the same limiting shape. This hints at the possibility of universality that does not exist in the case of isotropic surface tension (i.e. $v_{m}=0$ ).

\section{Boundary integral method with time and space rescaling}

In order to fully characterize the nonlinear morphological stability of a growing crystal, we use a spectrally accurate $2 \mathrm{D}$ boundary integral method in which a novel time and space rescaling is implemented [5]. This enables us to accurately simulate the long-time, nonlinear dynamics of evolving crystals. For completeness, the method is briefly described below.

Following [2,5], we introduce the spatial and temporal scaling of the dynamical equations

$\mathbf{x}=\bar{R}(\bar{t}) \overline{\mathbf{x}}(\bar{t}, \alpha)$,

$\bar{t}=\int_{0}^{t} \frac{1}{R\left(t^{\prime}\right)^{3}} \mathrm{~d} t^{\prime}$,

where $\bar{R}(\bar{t})=R(t(\bar{t}))$ and $\overline{\mathbf{x}}(\bar{t}, \alpha)$ is the position vector of the scaled interface, and $\bar{t}$ is the new time variable. The scaling $\bar{R}$ is chosen such that the area $\bar{A}$ enclosed by the scaled interface is constant in time. The scaling
$\bar{R}$ can be found by integrating the normal velocity over the interface and dividing by $2 \pi$ to get

$J=\frac{\bar{A}}{\pi} \frac{1}{\bar{R}^{2}(\bar{t})} \frac{\mathrm{d} \bar{R}(\bar{t})}{\mathrm{d} \bar{t}}$.

The normal velocity in the new frame is $\bar{V}(\bar{t}, \alpha)=$ $\frac{\mathrm{d} \overline{\mathbf{x}}(\bar{t}, \alpha)}{\mathrm{d} \bar{t}} \cdot \mathbf{n}$ and satisfies

$-\tau(\mathbf{n}) \bar{\kappa}-\bar{J} \mathcal{G}[\bar{x}]=\int_{\bar{\Sigma}} G\left(\left|\overline{\mathbf{x}}-\overline{\mathbf{x}}^{\prime}\right|\right) \bar{V} \mathrm{~d} s^{\prime}+\bar{T}_{\infty}(\bar{t})$,

and

$0=\int_{\bar{\Sigma}} \bar{V} \mathrm{~d} s$,

where $\bar{J}=\frac{\pi \bar{R} J}{\bar{A}}=\frac{\mathrm{d}}{\mathrm{d} \bar{t}} \log (\bar{R}(\bar{t}))$ and $\bar{\kappa}=\bar{R} \kappa$. Thus, the scaling factor is

$\bar{R}(\bar{t})=\exp \left(\int_{0}^{\bar{t}} \bar{J} \mathrm{~d} \bar{t}^{\prime}\right)$.

Further, in Eq. (19) we have taken $\bar{T}_{\infty}(\bar{t})=$ $\frac{\bar{A} \log (\bar{R})}{\pi} \bar{J}+T_{\infty}(t(\bar{t})) \bar{R}(\bar{t})$, and $\mathcal{G}(\overline{\mathbf{x}})=\int_{\bar{\Sigma}} \overline{\mathbf{x}}^{\prime} \cdot \mathbf{n}\left(\overline{\mathbf{x}}^{\prime}\right)$ $G\left(\overline{\mathbf{x}}-\overline{\mathbf{x}}^{\prime}\right) \mathrm{d} s^{\prime}$.

To evolve the interface numerically, Eqs. (19) and (20) are discretized in space using spectrally accurate discretizations and a scaled (equal arclength) parametrization [32]. The resulting discrete system is solved efficiently using GMRES together with a diagonal preconditioner in Fourier space [32,34]. Once $\bar{V}$ is obtained, the interface is evolved by using a second order accurate non-stiff updating scheme in time $[32,33]$.

\section{Isotropic results}

In this section, we briefly review our studies of a crystal with isotropic surface tension [5]. In [5], we applied fluxes of the form $J=C / R(t)$ in the far-field, where the flux constant $C$ is varied. As seen from Eq. (10) this choice of flux implies that the number of linearly unstable modes is bounded independent of the crystal size and the Mullins-Serkeka instability is suppressed.

Our analysis and simulations revealed that there is nonlinear stabilization leading to the growth of 
compact crystals even though unstable growth may be significant at early times. We observed three types of behavior: universal, limiting and oscillatory as discussed in Section 1. Universal behavior occurs when the long time shape of the evolving crystal is independent of both time and the initial shape. Limiting behavior occurs when the long time shape of the crystal is independent of time, but depends on the initial shape. Finally, oscillatory behavior occurs when the long time shape depends on both time and the initial shape, though its growth is bounded.

By performing a series of simulations, we constructed a limiting shape phase diagram that maps the limiting morphologies onto a graph shown in Fig. 1(a) in terms of their symmetries and associated flux constants $C$. Note that Fig. 1(a) is a simplified phase diagram, and the full version was shown in [5]. The set of dynamically achievable shapes is found to be in region II. The curve marking lower bound of region II describes the parameters appropriate to obtain universal shapes. Note that the universal shapes are determined solely by the heat flux. This curve takes a stair-case pattern because there are morphology transitions from $k$-fold to $(k+1)$-fold symmetries. The circles mark the transitions. Near these transitions, oscillatory behavior is observed. On the universal curve, the length of each step increases as the flux constant increases indicating that larger and larger fluxes are needed to produce higher and higher symmetries of the universal shapes. Note that because the surface energy is isotropic, the growing crystal may achieve any symmetry. There is a continuous set of flux constants that can be used to achieve a limiting (but not necessarily universal) shape of a particular symmetry. The shape factors $\delta / R$ of these limiting shapes are different. The shape factor $\delta / R$ measures the deviation from a circle and is defined as

$\delta / R=\max _{\tilde{\mathbf{x}} \in \Sigma}\left(|\tilde{\mathbf{x}}| /\left|\tilde{R}_{\text {eff }}-1\right|\right)$,

where $\tilde{\mathbf{x}}$ is the position vector from the centroid of the shape to the interface and $\tilde{R}_{\text {eff }}$ is the effective radius of the shape.

In Fig. $1[\mathrm{~b}]$ we examined the relation between the shape factors $\delta / R$ of the universal limiting shapes and the flux constant. The vertical dashed lines indicate transitions from symmetry $k$ to $k+1$. Representative
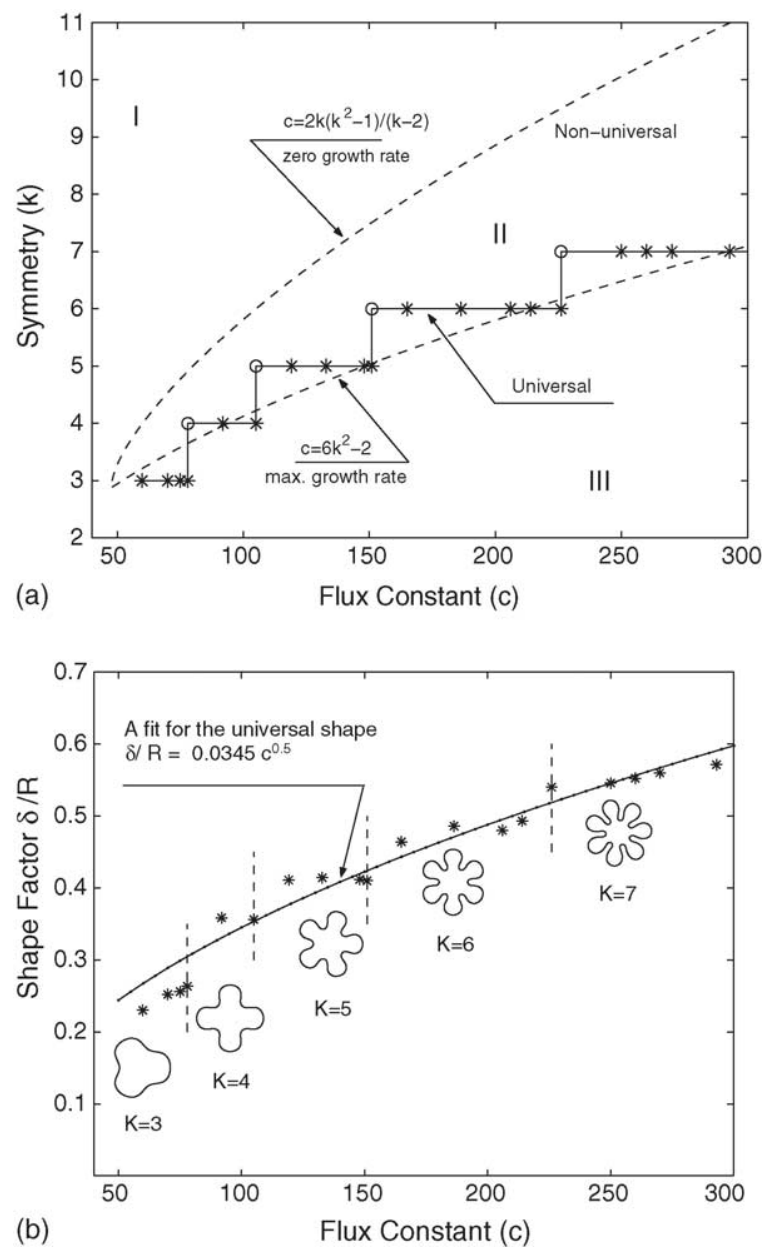

Fig. 1. (a) Limiting shape phase diagram. The set of dynamically achievable shapes is found to be region II. Region II is bounded by an upper dashed curve (linear theory) and a piecewise constant curve (nonlinear results) that describe the relation between the observed symmetries of the limiting shape and the flux constant. The stars denote actual simulation data. The universal limiting shapes lie on the lower piecewise constant curve. In between these curves, non-universal shapes may occur with finite multiplicity. The upper dashed curve shows the relation between the flux constant and the mode with zero growth rate (i.e. self-similar). The lower dashed curve shows the relation between the flux constant and the fastest growing mode. (b) The associated shape factors of the universal limiting shapes. The solid-dotted curve suggests that the asymptotic behavior may be $\delta / R \sim \sqrt{C}$ at large flux constants $C$. The vertical dashed lines indicate transitions from symmetry $k$ to $k+1$. Representative universal morphologies are shown for each symmetry. 
universal morphologies are shown for each symmetry. The solid-dotted curve suggests that the asymptotic behavior may be $\delta / R \sim \sqrt{C}$ at large flux constants $C$. This suggests the intriguing possibility there may be a critical value of $C$ at which the topology of the universal shape changes from a simply connected to a multiply connected region characterized by a shape with identical, equally spaced fingers that are all connected at the origin (i.e. there is an inner tangent circle with vanishing radius). This is currently under study.

\section{Anisotropic results}

In this section, we present the results of a parameter study in which the surface tension anisotropy is four-fold symmetric, i.e. $m=4$ in Eq. (6), and the anisotropy strength $v=v_{4}$ is varied. Because we want the growth and surface tension time scales to balance, we use fluxes of the form $J=C / R(t)$, where the flux constant $C$ is also varied.

Our analysis and simulations reveal that, as in the isotropic case, nonlinearity stabilizes the evolution of a growing crystal even though unstable growth may be significant at early times. In particular, we observe three types of behavior: universal, limiting and oscillatory. By performing simulations with a variety of initial shapes, anisotropy and specified flux constants, we map the resulting morphologies onto a phase-diagram that is shown in Fig. 2(a). In this figure, the parameter space is accordingly divided into three regions according to the results of long-time simulations. The stars are a result of simulations that define the boundaries of the regions up to an accuracy of approximately $5 \%$ in the flux constant $C$. The other symbols are the results of specific simulations that will be discussed later.

The set of the dynamically achievable universal limiting shapes found in this paper is contained in the region marked I. That is, for each value of $v$ and $C$ in region I, the evolution tends to a unique four-fold symmetric universal shape. The four-fold symmetry is due to the anisotropy and the fact that the universal shape is independent of the initial data. This is in contrast to the isotropic case we studied previously where universal shapes are achievable with any symmetry [5] and depend only on the flux constant. Here, the surface ten-
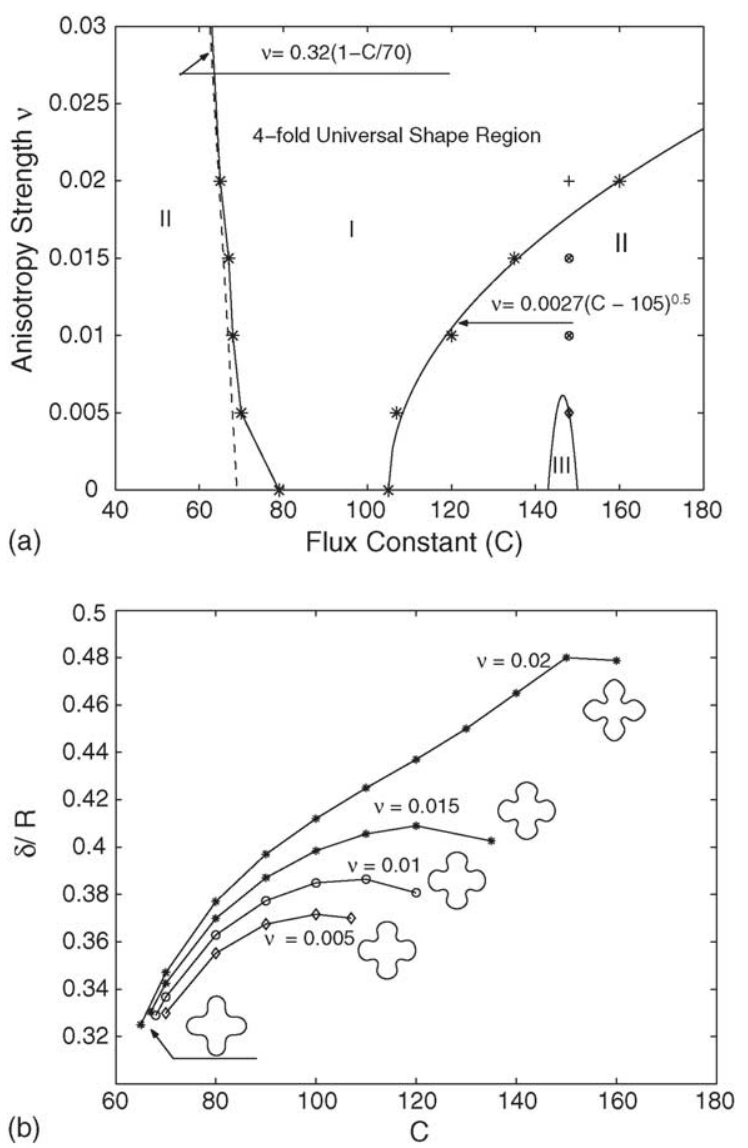

Fig. 2. (a) Limiting shape phase diagram. Region I: The set of dynamically achievable universal shapes. Region II: the set of dynamically initial-data dependent limiting shapes. Region III: the set of limiting and oscillatory shapes. (b) The associated shape factors of the universal limiting shapes for different anisotropy strengths $v$.

sion anisotropy selects the symmetry of the universal shape.

Region I is bounded on the left by a nearly linear curve which is qualitatively consistent with linear theory. This is seen as follows. From Eq. (13) linear theory predicts that the flux for which mode 4 is the fastest growing mode is a decreasing function of $v$. If in Eq. (13) we set $l=m=4$ and replace the left hand side by $C / R(t)$ and the right hand side by $C_{4}^{*} / R(1-v) /(\delta / R)$ then we can solve for $v$ to obtain $v=\delta / R\left(1-C / C_{4}^{*}\right)$ which indicates a linear dependence of $v$ on the flux constant $C$. The curve shown in the figure is the result of using a characteristic value $\delta / R$ obtained from the 
nonlinear simulations and fitting $C_{4}^{*}$ to the nonlinear data (instead of taking $C_{4}^{*}=94$ as predicted by linear theory (e.g. see Eq. 13)). In the figure, we take $\delta / R=0.32$ and $C_{4}^{*}=70$.

According to linear theory, the range of flux constants $C$ for which mode 4 is dominant is $94=C_{4}^{*} \leq$ $C<C_{4}^{* *}$ where $C_{4}^{* *}$ is the flux constant such that mode 4 and mode 5 have the same growth rate. A calculation shows that the relationship $C=C_{4}^{* *}$ from linear theory can be solved for $v$ in terms of $C$. It follows that $v$ is an increasing function of $C$ consistent with the nonlinear results. In this sense, surface tension anisotropy has a stabilizing effect on the evolution.

In regions II and III non-universal behavior is observed. Region II consists of two subregions. The first subregion is on the low flux side of the phase diagram, and its boundary with region $I$ is at a flux constant of approximately 70 . The boundary with region I is only weakly dependent on $v$. In this subregion the limiting shapes have symmetries dominated by modes 3 or 4 , depending on the initial conditions. The second subregion is on the high flux side of the phase diagram, and its boundary with region I depends strongly on $v$. As expected in this region the limiting shapes have symmetries dominated by modes 5 and higher.

Interestingly, we also find a small region III in which the evolution exhibits oscillatory behavior at long times and the growth shapes do not tend to a limiting shape although the growth is bounded. The oscillatory behavior is inherited from the isotropic case. In the isotropic case, oscillatory behavior is observed when the flux constant is near the transition regions separating mode $k$-dominated growth from mode $k+1$-dominated growth (see also [5]). The oscillatory behavior observed in region III here is due to the transition that occurs in the isotropic case between five-fold and six-fold symmetric universal shapes $(C \approx 148)$. For flux constants away from the transition region, oscillatory behavior is not observed. Moreover, as $v$ increases, region III narrows. This also suggests a stabilizing effect of surface tension anisotropy in the sense that the anisotropy limits the growth directions.

In Fig. 2[b], we present the shape factors $\delta / R$ associated with the universal limiting shapes for different anisotropy strengths $v$ and flux constants $C$. Recall that the shape factor measures the deviation from a circle.
For a crystal with anisotropic surface tension, the shape factor $\delta / R$ is a nonlinear function of both $v$ and $C$.

The shape factor is an increasing function of $v$ and is non-monotone in $C$ owing to the change in symmetry of the limiting shape as it passes from region I (universal) to region II. In all cases $\delta / R$ is quite large indicating that nonlinearities are important. Note that when $C$ is small, the shape factor depends only weakly on $v$. This is currently under study.

To illustrate the range of behavior described above in regions I, II and III, we consider a case in which the flux constant is fixed $C=148$ and the anisotropy $v$ is decreased from $v=0.02$ to 0.005 . These parameters are chosen so that the shapes transit from region I to region II to region III (e.g. see the plus, cross and diamond symbols in Fig. 2(a)). In Fig. $3(\mathrm{a})$, we take $v=0.02$ and plot the evolution of the shape factor $\delta / R$ as a function of $R$ for three different initial conditions. This corresponds to region I. This Figure shows that although there is significant growth of the perturbations at early times, linear theory overpredicts the growth. The simulations show that there is strong nonlinear stabilization and the shape factors converge to a single value that characterizes the universal shape. The associated crystal morphologies are shown as insets. Note that the evolution from the initial data that does not contain mode 4 takes a relatively longer time (larger $R$ ) to converge to the universal shape since mode 4 must be generated by the anisotropy and nonlinear interactions among modes. By performing an analysis similar to that presented in [5], we can demonstrate that indeed the universal shape is a nonlinear self-similar solution of the crystal growth equations [4].

We next decrease the anisotropy strength to $v=$ 0.01 keeping the flux constant $C=148$. This corresponds to region II. The resulting plot of $\delta / R$ versus $R$ is shown in Fig. 3(b) for initial shapes $r(0)=1.0+0.01(\cos 4 \alpha+\cos 5 \alpha)$ and $r(0)=1.0+$ $0.01(\cos 2 \alpha+\cos 3 \alpha+\cos 5 \alpha+\cos 6 \alpha)$. The associated crystal morphologies are shown as insets. The perturbations grow significantly at early times and the evolution of the initial shape $r(0)=1+0.01(\cos 4 \alpha+$ $\cos 5 \alpha$ ) yields a mode 5 dominant shape at its early times (small $R$ ). As $R$ increases, oscillations appear in the shape factor due to a complex process of tip- 
splitting. Eventually, mode 6 emerges and the evolution tends toward a six-fold limiting shape. However, for the case with initial data $r(0)=1+0.01(\cos 2 \alpha+$ $\cos 3 \alpha+\cos 5 \alpha+\cos 6 \alpha$ ), the evolving shape is al-
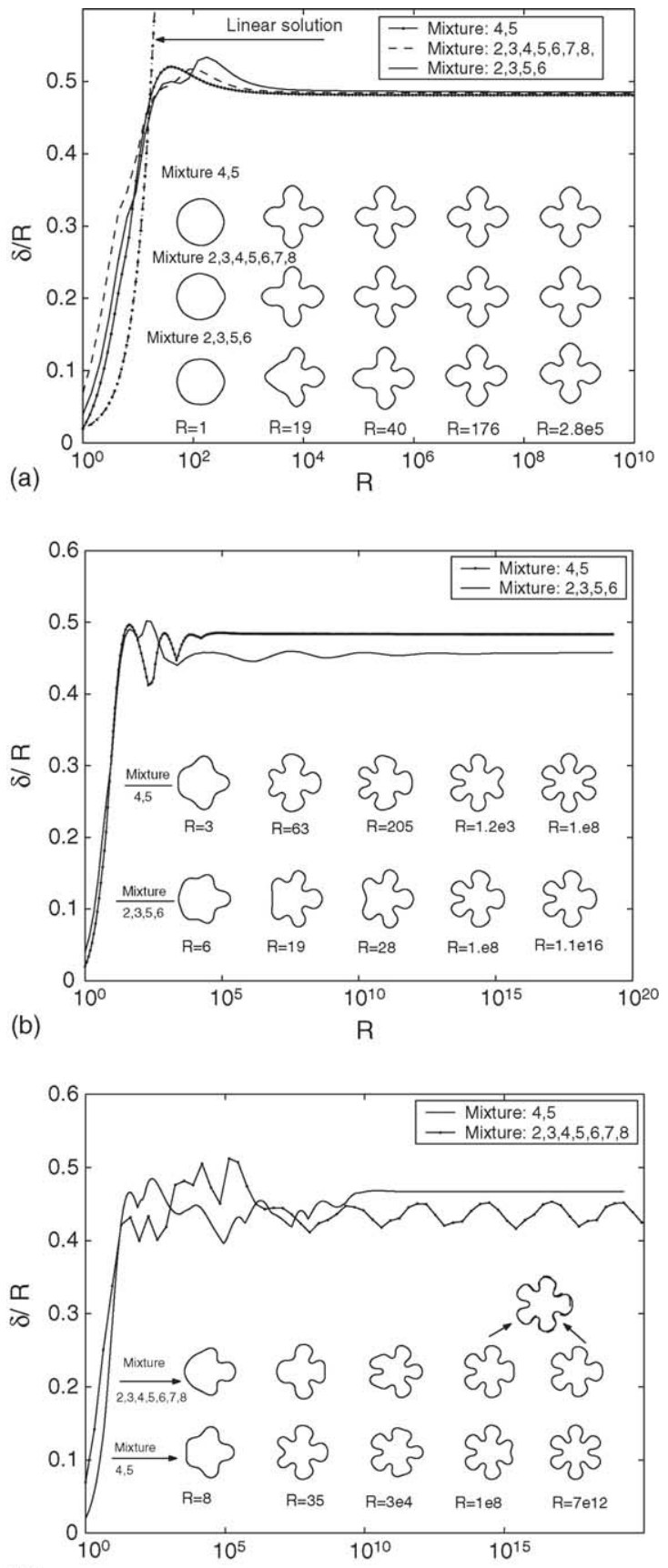

(c)

$\mathrm{R}$ ways 5 mode dominant and converges to a limiting shape at very large $R$. By performing an analysis similar to that presented in [5], we can demonstrate that both limiting shapes obtained here are nonlinear selfsimilar solutions. This implies that there is no universal shape when $v=0.01$ and $C=148$.

We next decrease the anisotropy strength to $v=0.005$. This corresponds to region III. The results are shown in Fig. 3(c) for two different initial data. The evolution of the initial data $r(0)=1+0.01(\cos 2 \alpha+$ $\cos 3 \alpha+\cos 4 \alpha+\cos 5 \alpha+\cos 6 \alpha+\cos 7 \alpha+$ $\cos 8 \alpha$ ) is oscillatory and the growth of the shape factor is bounded. The associated morphologies are shown as insets. The last two shapes from a valley and peak, corresponding to $R=10^{8}, 7 \times 10^{12}$, respectively, of the oscillatory curve $\delta / R$ are compared. The figure indicated by the two arrows shows the morphologies of the last two shapes plotted on top of one another. The difference between the two shapes is clearly seen. Note that the differences in these two shapes are primarily confined to a small region near the rightmost finger. It appears that this finger fails to tip-split to form a 6-mode dominated shape and instead oscillates. In contrast, the evolution of the initial data $r(0)=1+0.01(\cos 4 \alpha+\cos 5 \alpha)$ tends toward a six-fold symmetric limiting shape at very large $R$.

Fig. 3. (a) Shape factor evolution is shown for three arbitrary initial shapes, $r(0)=1.0+0.01(\cos 4 \alpha+\cos 5 \alpha), r(0)=1.0+$ $0.01(\cos 2 \alpha+\cos 3 \alpha+\cos 4 \alpha+\cos 5 \alpha+\cos 6 \alpha+\cos 7 \alpha+$ $\cos 8 \alpha), \quad$ and $\quad r(0)=1.0+0.01(\cos 2 \alpha+\cos 3 \alpha+\cos 5 \alpha+$ $\cos 6 \alpha$ ). The anisotropy strength is set to be $v=0.02$. The associated morphologies are shown. The far-field flux is taken to be $J=148 / R(t)$. The evolution tends to a universal limiting shape with a 4 -fold symmetry. The dashed-dot line is the linear theory prediction for the mode mixture of 4 and 5. (b) Non-universal shape evolution when the far-field flux $J=148 / R(t)$ and anisotropy strength $v=0.01$. The shape starting from a mixture $r(0)=1.0+0.01(\cos 4 \alpha+\cos 5 \alpha)$ tends to a 6-mode dominant limiting shape. The shape starting from a mixture $r(0)=1.0+0.01(\cos 2 \alpha+\cos 3 \alpha+\cos 5 \alpha+\cos 6 \alpha)$ evolves to a 5-mode dominant limiting shape. (c) Non-universal shape evolution when the far-field flux $J=148 / R(t)$ and anisotropy strength $v=0.005$. The shape starting from a mixture $\quad r(0)=1.0+0.01(\cos 2 \alpha+\cos 3 \alpha+\cos 4 \alpha+\cos 5 \alpha+$ $\cos 6 \alpha+\cos 7 \alpha+\cos 8 \alpha)$ shows an oscillatory behavior and is 5-mode dominant. The shape starting from a mixture $r(0)=1.0+0.01(\cos 4 \alpha+\cos 5 \alpha)$ tends to a 6-mode dominant limiting shape. 


\section{Conclusion and discussion}

In this paper, we characterized the nonlinear morphological stability of 2D anisotropic crystals using a spectrally accurate 2D boundary integral method with a novel time and space rescaling scheme developed in [5]. We simulated the long-time, nonlinear dynamics of evolving crystals. Our analysis and simulations revealed that as in the isotropic case nonlinearity stabilized the evolution of a growing crystal even though unstable growth was significant at early times. The crystal morphologies were determined by a complex competition between the heat transport (far-field flux) and surface tension anisotropy. As in the isotropic case, we observed three types of behavior: universal, limiting and oscillatory. The results of a parameter study were mapped onto a phase-diagram that separates the parameter space into three regions that are characterized by these three types of evolution.

The phase-diagram presented here can be used to design a nonlinear protocol that might be able to be carried out in a physical experiment to control the nonlinear morphological evolution of a crystal as follows. For a given value of surface tension anisotropy, the phase-diagram yields the range of fluxes such that an arbitrarily shaped crystal will evolve to a universal limiting shape. The symmetry of the universal shape is consistent with the symmetry of the surface tension anisotropy. The shape factor of the universal shape is a nonlinear function of the heat flux and anisotropy and is also determined from our simulations. For example, the size of the perturbation can be controlled by a careful selection of $C$. In addition, the evolution towards the universal shape is faster for choices of $C$ away from the boundary of region I. In an experiment, the flux condition can be imposed by varying the far-field temperature in time following Eq. (9).

Even though this paper focused on four-fold symmetric surface energy anisotropies, we have performed studies for other anisotropy symmetries and found similar results. Thus our results apply more generally.

While this paper focused on crystal growth in 2D, our analysis applies qualitatively to $3 \mathrm{D}[4,5]$. We are currently performing 3D simulations to confirm that our 2D model indeed captures the significant features of 3D growth. In future work, we will demonstrate that universal attractive 3D limiting shapes exist.

Our results show that in some cases the evolution towards universal limiting shapes may be somewhat slow with the universal shape being achieved only when the crystal grows very large compared with its initial size. The rate of convergence to the universal limiting shapes is initial data-dependent even though the universal shape itself is independent of the initial data. For instance, the evolution towards the universal shape is faster starting from initial shapes containing many modes. As mentioned above, a careful selection of $C$ can also accelerate the growth process.

Depending on the initial size of the crystal, gravitational effects may become important during the evolution. This can generate effective fluid motion and convection. This potentially important physical effect is neglected here. We have also neglected other important physical effects such as interface kinetics. These effects can play a significant role in the development of the complex patterns seen during crystal growth. We are currently investigating these physical effects. For example, our results indicate that nonlinear, stable, self-similar shapes exist for finite interface kinetics as well [37].

Finally, the analysis developed here may provide insights on the control of pattern formation in other physical and biological systems.

\section{Acknowledgements}

The authors thank Professors Vittorio Cristini and Robert Sekerka for stimulating discussions. S. Li is supported by a Doctoral Dissertation Fellowship from the Graduate School, University of Minnesota. The authors also acknowledge the generous computing resources from the Minnesota Supercomputer Institute (MSI), the Network and Academic Computing Services at University of California at Irvine (NACS), and computing resources of the BME department (U.C. Irvine). S. Li also thanks the support from the Department of Biomedical Engineering and the Department of Mathematics at U.C. Irvine where he is a visiting researcher. J. Lowengrub thanks the National Science Foundation (Division of Mathematical Sciences) for partial support. 


\section{References}

[1] E. Ben-Jacob, P. Garik, The formation of patterns in nonequilibrium growth, Nature 343 (1990) 523.

[2] V. Cristini, J. Lowengrub, Three-dimensional crystal growth. I: linear analysis and self-similar evolution, J. Cryst. Growth 240 (2002) 267-276.

[3] V. Cristini, J. Lowengrub, Three-dimensional crystal growth. II: nonlinear simulation and control of the Mullins-Sekerka instability, J. Cryst. Growth 266 (2004) 552-567.

[4] S. Li, J. Lowengrub, P. Leo, V. Cristini, nonlinear theory of self-similar crystal growth and melting, J. Cryst. Growth 267 (2004) 703-713.

[5] S. Li, J. Lowengrub, P. Leo, V. Cristini, Nonlinear stability Analysis of self-similar crystal growth: control of MullinsSekerka instability, J. Cryst. Growth 277 (2005) 578-592.

[6] J.S. Langer, Instability and pattern formation in crystal growth, Rev. Modern Phys. 52 (1980) 1.

[7] G.B. McFadden, S.R. Coriell, R.F. Sekerka, Effect of surface free energy anisotropy on dendrite tip shape, Acta Mater. 48 (2000) 3177-3181.

[8] G.B. McFadden, S.R. Coriell, R.F. Sekerka, Analytic solution for a non-axisymmetric isothermal dendrite, J. Cryst. Growth 208 (2000) 726-745.

[9] D.M. Anderson, G.B. McFadden, A.A. Wheeler, A phase-field model with convection: sharp interface asymptotics, Physica D 151 (2001) 305-331.

[10] T. Uehara, R.F. Sekerka, Phase field simulations of faceted growth for strong anisotropy of kinetic coefficient, J. Cryst. Growth 254 (2003) 251-261.

[11] W.W. Mullins, R.F. Sekerka, Stability of a Planar Interface During Solidification of a Dilute Binary Alloy, J. Appl. Phys. 35 (2) (1964) 444-451.

[12] L.N. Brush, R.F. Sekerka, A numerical study of twodimensional crystal growth forms in the presence of anisotropic growth kinetics, J. Cryst. Growth 96 (1989) 419441.

[13] S.R. Coriell, R.F. Sekerka, The effect of the anisotropy of surface tension and interface kinetics on morphological stability, J. Cryst. Growth 34 (1976) 157-163.

[14] Y. Saito, Statistical Physics of Crystal Growth, World Scientific, Singapore, 1996.

[15] W.W. Mullins, R.F. Sekerka, Morphological stability of a particle growing by diffusion or heat flow, J. Appl. Phys. 34 (2) (1963) 323-329.

[16] S.R. Coriell, R.L. Parker, Stability of the shape of a solid cylinder growing in a diffusion field, J. Phys. 36 (1965) 632637.

[17] S.R. Coriell, R.L. Parker, H.S. Peiser (Eds.), Crystal Growth, Pergamon, Oxford, 1967, p. 703.
[18] A.A. Golovin, S.H. Davis, Effect of anisotropy on morphological instability in the freezing of a hypercooled melt, Physica D 116 (1998) 363.

[19] B. Li, A. Ratz, A. Voigt, Stability of a circular epitaxial island, Physica D 198 (2004) 231.

[20] D.A. Kessler, H. Levine, Microscopic selection of fluid fingering patterns, Phys. Rev. Lett. 86 (2001) 4532.

[21] E. Ben-Jacob, I. Cohen, H. Levine, Cooperative selforganization of microorganisms, Adv. Phys. 49 (2000) 395.

[22] V. Cristini, J. Lowengrub, Q. Nie, Nonlinear simulation of tumor growth, J. Math. Biol. 46 (2003) 191.

[23] X. Zheng, S.M. Wise V. Cristini, Nonlinear simulation of tumor necrosis, neo-vascularization and tissue invasion via an adaptive finite-element/level-set method, Bull. Math. Biol. 67 (2005) 259.

[24] P. Macklin, J. Lowengrub, Evolving interfaces via gradients of geometry-dependent interior Poisson problems: application to tumor growth, J. Comp. Phys. 203 (2005) 191.

[25] S.C. Hardy, S.R. Coriell, Morphological stability of a cylinder, J. Res. Bureau Standards-A. Phys. Chem. 73A (1) (1969) 6568.

[26] M.E. Glicksman, A. Lupulescu, M.B. Koss, Melting in Microgravity, J. Thermophys. Heat Transfer 17 (1) (2003) 69-76.

[27] M.E. Glicksman, R.J. Shaefer, J.D. Ayers, Dendritic growth-a test of theory, Metall Trans. A 7A (1976) 1747-1759.

[28] M.E. Glicksman, M.B. Koss, E.A. Winsa, Dendritic growth velocities in microgravity, Phys. Rev. Lett. 73 (1994) 573.

[29] J.C. LaCombe, M.B. Koss, M.E. Glicksman, nonconstant tip velocity in microgravity Dendritic growth, Phys. Rev. Lett. 83 (1999) 2997-3000.

[30] J. Alkemper, R. Mendoza, P.W. Voorhees, Morphological evolution of Dendritic microstructures, Advanced Eng. Mater. 4 (2002) 694-697.

[31] J. Alkemper, P.W. Voorhees, Three-dimensional characterization of Dendritic microstructures, Acta Mater. 49 (2001) 897902.

[32] T.Y. Hou, J.S. Lowengrub, M.J. Shelley, Boundary integral methods for multicomponent fluids and multiphase materials, J. Comp. Phys. 169 (2) (2001) 302-362.

[33] T.Y. Hou, J.S. Lowengrub, M.J. Shelley, Removing the stiffness from interfacial flows with surface tension, J. Comp. Phys. 114 (1994) 312.

[34] H.J. Jou, P. Leo, J. Lowengrub, Microstructural evolution in inhomogeneous elastic media, J. Comp. Phys. 131 (1997) 109148.

[35] S. Osher, B. Merriman, The wulff shape as the asymptotic limit of a growing crystalline interface, Asian J. Math. 1 (1997) 560.

[36] G. Wulff, Z. Kristallogr, Mineral. 34 (1901) 449.

[37] S. Li, Morphological control of crystal growth, Ph.D. 2005 expected, University of Minnesota. in press. 